\title{
Maintaining cerebral oxygen homeostasis: a serious business
}

\author{
Andreas Koch ${ }^{1}$ - Wataru Kähler ${ }^{1}$ - Sebastian Klapa ${ }^{1}$ · Johann P. Kuhtz-Buschbeck ${ }^{2}$
}

Received: 25 May 2018 / Accepted: 26 May 2018 / Published online: 6 June 2018

c) Springer-Verlag GmbH Germany, part of Springer Nature 2018

Studies under extreme physiological conditions can be very enlightening to understand the organism's mechanisms for maintaining homeostasis, and, in particular, an adequate oxygen delivery to the organs and tissues. Several circumstances can challenge oxygen homeostasis. For instance, ascent to high altitude may jeopardize proper body oxygen supply; diving with oxygen may result in non-physiological hyperoxia; and circulatory failure may result in organ dysfunction, therefore triggering compensatory physiological reactions in order to restore homeostasis.

In this issue of Clinical Autonomic Research, Hoiland, Ainslie and colleagues, from the University of British Columbia, [1] give fundamental insights into the adaptive processes required to keep adequate oxygen delivery to the central nervous system under hypoxic ambient conditions that occur at high altitude. Acute hypoxia after ascent, when complete acclimatization is not yet present, requires a rapid adaptation of the autonomic nervous system in order to prevent acute cerebral malfunction.

After acclimatization, long-lasting residence at high altitude results in a number of less rapid adaptations. These, including increase in blood hemoglobin levels, lead to a stepwise reduction in the need for acute autonomic regulation, as these are no longer required to maintain the physiological oxygen supply homeostasis. Such a stepwise reduction of an acute regulatory process was thoroughly described in the manuscript by Hoiland, Aislie and colleagues. In particular, their description of the cerebral blood flow regulation, the comparison between the development of arterial oxygen

This is a comment on an article available at: https://link.springer. com/article/10.1007/s10286-018-0522-2.

Andreas Koch

a.koch@iem.uni-kiel.de

1 Naval Institute of Maritime Medicine, Section for Maritime Medicine of the Christian-Albrechts-University, Kiel, Germany

2 Institute of Physiology, Christian-Albrechts-University, Kiel, Germany content together with the hypoxic ventilatory response and the subsequent changes in cerebral blood flow, in the light of a constantly maintained oxygen delivery to the central nervous system in the process of high-altitude adaption over 2 weeks, was enlightening. This synopsis of data from a number of different studies shows that the initially increased cerebral blood flow returns to normal values as arterial oxygen levels increase due to hyperventilation, hemoconcentration and subsequent rising hematocrit. These compensatory mechanisms gradually make increased cerebral perfusion unnecessary.

But what happens under the exact opposite situation, i.e., breathing $100 \%$ oxygen or even hyperbaric pure oxygen? Surprisingly, this very unphysiological situation causes a reduction in cerebral blood flow of $\sim 10-15 \%[2,3]$, which is sometimes harnessed in neurosurgical intensive care in order to reduce cerebral edema. However, whereas the need for increased cerebral flood flow at high altitude (which can last for up to 2 weeks) is obvious, an active reduction of the cerebral blood flow under hyperoxia is not that intuitive. The answer for this phenomenon is not clear, but the most accepted hypothesis is primarily based on a decrease in available local endothelial nitric oxide (NO) caused by inhibition of NO-synthesis, production of peroxynitrite $\left(\mathrm{ONOO}^{-}\right)$and reduced release from S-nitrosohemoglobin [4]. Under these circumstances, the delivery of oxygen to the central nervous system remains unimpaired, in spite of reduced cerebral blood flow.

In addition to high altitude, acute compensatory increases in cerebral blood flow to maintain cerebral oxygen homeostasis also occur in other circumstances characterized by sudden drops in blood pressure and heart rate, such as neurocardiogenic-vasovagal-syncope, or right after stopping strenuous muscular effort [5]. In the case of vasovagal syncope, the autonomic nervous system not only attempts to rapidly stabilize cerebral perfusion (as measured by transcranial Doppler ultrasound) after the syncopal event, but even increases cerebral blood flow dramatically as to temporarily exceed resting values $[6,7]$. A very similar reaction can be observed after stopping a hard muscular effort 
without proper cooling down, when blood pressure and heart rate rapidly drop down to normal resting levels, without decreasing further as to reach hypotension or bradycardia [6]. During strenuous muscular work, cerebral blood flow increases $\sim 15 \%$ and stabilizes, whereas blood pressure and heart rate show the typical pattern during exercise regulation. Right after stopping, however, a sudden autonomic reaction brings cerebral blood flow immediately back to the previous working state, before it slowly downregulates back to normal values within the next $2 \mathrm{~min}$, similar to what happens after a typical vasovagal syncope $[6,7]$.

Cerebral blood flow regulation is a key mechanism to maintain oxygen homeostasis, both during acute conditions (e.g., to stabilize cerebral perfusion during hypotension), and to ensure adequate oxygen delivery for a longer period of time at high altitude during acclimatization as elegantly illustrated by Hoiland and colleagues in their model about the opposing influences of ventilation and cerebral blood flow on the central chemoreceptors, which are decisively involved in the regulatory process.

Thus, maintaining an adequate oxygen delivery to the brain is a serious business, particularly so when a moderately decreased cerebral blood flow seems to be "tolerated" without significant regulatory countermeasures during sustained hyperoxia.

\section{Compliance with ethical standards}

Conflict of interest The authors declare that they have no conflict of interest.

\section{References}

1. Hoiland RL, Howe CA, Coombs GB, Ainslie PN (2017) Ventilatory and cerebrovascular regulation and integration at high-altitude. Clin Auton Res. https://doi.org/10.1007/s10286-018-0522-2

2. Koch AE, Koch I, Kowalski J, Schipke JD, Winkler BE, Deuschl G, Meyne J, Kähler W (2013) Physical exercise might influence the risk of oxygen-induced acute neurotoxicity. Undersea Hyperb Med 40(2):155-163

3. Visser GH, Van Hulst RA, Wieneke GH, Van Huffelen AC (1996) Transcranial doppler sonographic measurements of middle cerebral artery flow velocity during hyperbaric oxygen exposures. Undersea Hyperb Med 23:157-165

4. Demchenko IT, Boso AE, O'Neill TJ, Bennett PB, Piantadosi CA (2000) Nitric oxide and cerebral blood flow responses to hyperbaric oxygen. J Appl Physiol 88:1381-1389

5. Koch A, Ivers M, Gehrt A, Schnoor P, Rump A, Rieckert H (2005) Cerebral autoregulation is temporarily disturbed in the early recovery phase after dynamic resistance exercise. Clin Auton Res 15(2):83-91

6. Edwards MR, Schondorf R (2003) Is cerebrovascular autoregulation impaired during neurally-mediated syncope? Clin Auton Res 13(5):306-309

7. Diehl RR, Linden D, Chalkiadaki A, Ringelstein EB, Berlit P (1996) Transcranial Doppler during neurocardiogenic syncope. Clin Auton Res Off J Clin Auton Res Soc 6(2):71-74 